\title{
PERFORMANCE OF STATISTICAL INFERENCE METHODS FOR THE ENERGY ESTIMATION OF MULTIPLE SOURCES
}

\author{
Romain Couillet ${ }^{1}$ and Maxime Guillaud ${ }^{2}$ \\ ${ }^{1}$ Chair "System Sciences and the Energy Challenge", Supélec, France, ${ }^{2}$ ITC, Vienna University of Technology, Austria.
}

\begin{abstract}
This article considers statistical inference of the transmit powers of multiple signal sources by a sensor network. Using random matrix methods, an exact expression of the posterior probability of the joint transmit powers is derived. This expression is used to implement the associated ML and MMSE detectors of the joint powers. These are compared for small system sizes against an asymptotically unbiased estimator obtained from large dimensional random matrix theory.
\end{abstract}

\section{INTRODUCTION}

Several works have recently emerged which use large dimensional random matrix theory in order to address array processing problems and in particular statistical inference, see e.g. [1], [2], [3]. Large dimensional random matrix theory has often proven to provide compact and computationally simple results to deal with multi-variate problems in wireless communications, many examples of which are detailed in [4]. Nonetheless, few results are available that evaluate the performance of these methods for signal processing purposes. In this article, we consider a simple problem of multiple source power estimation for which [2] provides a large random matrix estimator but which we address here from an optimal finite dimensional point of view in order to compare the performance of optimal maximum likelihood (ML) and minimum mean square error (MMSE) estimators to the performance of the large dimensional estimator. Conversely to [2], the approach proposed here is not handicapped by any cluster separability condition.

\section{POSITION OF THE PROBLEM}

We consider the scenario of $K$ signal sources, source $k$ being equipped with $n_{k}$ collocated antennas. A sensor network composed of $N$ sensors captures the data originating from the $K$ sources in $M$ consecutive time samples $\mathbf{y}_{1}, \ldots, \mathbf{y}_{M} \in \mathbb{C}^{N}$, collected in the matrix $\mathbf{Y}=\left[\mathbf{y}_{1}, \ldots, \mathbf{y}_{M}\right] \in \mathbb{C}^{N \times M}$. The power of source $k$ received at the sensor array is denoted $P_{k}$. Denoting $\mathbf{H}_{k} \in \mathbb{C}^{N \times n_{k}}$ the Gaussian channel between source $k$ and the sensor array (constant during the $M$ observations), with entries of zero mean and variance $1 / N$, we then have the communication model

$$
\mathbf{Y}=\sum_{k=1}^{K} \sqrt{P_{k}} \mathbf{H}_{k} \mathbf{X}_{k}+\sigma \mathbf{W}
$$

with $\mathbf{X}_{k} \in \mathbb{C}^{n_{k} \times M}$ formed by the $M$ successive data vectors transmitted by source $k$, assumed Gaussian with entries of zero mean and unit variance and $\mathbf{W}$ the receive AWGN matrix with entries of zero mean and unit variance. Writing $\mathbf{H} \triangleq$ $\left[\mathbf{H}_{1}, \ldots, \mathbf{H}_{K}\right] \in \mathbb{C}^{N \times n}, \mathbf{X}=\left[\mathbf{X}_{1}^{\top}, \ldots, \mathbf{X}_{K}^{\top}\right]^{\top} \in \mathbb{C}^{n \times M}$ and $\mathbf{P}=\operatorname{diag}\left(P_{1}, \ldots, P_{1}, P_{2}, \ldots, P_{2}, \ldots, P_{K}, \ldots, P_{K}\right)$ with $P_{k}$ of multiplicity $n_{k}$ and where $n \triangleq \sum_{k=1}^{K} n_{k}$, we finally have

$$
\mathbf{Y}=\left[\begin{array}{ll}
\mathbf{H P}^{\frac{1}{2}} & \sigma \mathbf{I}_{N}
\end{array}\right]\left[\begin{array}{c}
\mathbf{X} \\
\mathbf{W}
\end{array}\right]
$$

We further denote $\mathbf{R}$ the covariance matrix of $\mathbf{y}_{m}$, conditionally on $\mathbf{H}$, i.e. $\mathbf{R}=\mathrm{E}\left[\mathbf{y}_{m} \mathbf{y}_{m}^{\mathrm{H}}\right]=\mathbf{H} \mathbf{P} \mathbf{H}^{\mathrm{H}}+\sigma^{2} \mathbf{I}_{N}$.

We seek an explicit expression for the posterior probability $P_{P_{1}, \ldots, P_{K} \mid \mathbf{Y}}(\mathbf{Y})$. From Bayes' rule, we have

$$
P_{P_{1}, \ldots, P_{K} \mid \mathbf{Y}}(\mathbf{Y})=P_{\mathbf{Y} \mid P_{1}, \ldots, P_{K}}(\mathbf{Y}) \frac{P_{P_{1}, \ldots, P_{K}}}{P_{\mathbf{Y}}(\mathbf{Y})}
$$

with

$$
\begin{array}{r}
P_{\mathbf{Y}}(\mathbf{Y})=\int_{\mathbb{R}_{+}^{K}} P_{\mathbf{Y} \mid P_{1}, \ldots, P_{K}}(\mathbf{Y}) P_{P_{1}, \ldots, P_{K}} d P_{1} \ldots d P_{K} \\
P_{\mathbf{Y} \mid P_{1}, \ldots, P_{K}}(\mathbf{Y})=\int_{\{\mathbf{R}>0\}} P_{\mathbf{Y} \mid \mathbf{R}, P_{1}, \ldots, P_{K}}(\mathbf{Y}) P_{\mathbf{R} \mid P_{1}, \ldots, P_{K}} d \mathbf{R} .
\end{array}
$$

The question here is to express explicitly the quantities $P_{\mathbf{Y} \mid \mathbf{R}}(\mathbf{Y})$ and $P_{\mathbf{R} \mid P_{1}, \ldots, P_{K}}$.

\section{POSTERIOR DISTRIBUTION OF $P_{1}, \ldots, P_{K}$}

We assume in the following that $n \leq N \leq M$ and we further denote $\mathbf{P}=\operatorname{diag}\left(p_{1}, \ldots, p_{n}\right)$, i.e. $p_{n_{1}+\ldots+n_{k-1}+1}=\ldots=$ $p_{n_{1}+\ldots+n_{k}}=P_{k}$ and define the mapping $\eta(i)$ to be such that $p_{i}=P_{\eta(i)}$.

Now, conditioned on $\mathbf{R}$, the entries of $\mathbf{Y}$ are the linear combination of Gaussian random variables with zero mean and unit variance. The matrix $\mathbf{Y} \in \mathbb{C}^{N \times M}$ is therefore Gaussian of zero mean and columns of covariance $\mathbf{R}$. Therefore we have

$$
\begin{aligned}
P_{\mathbf{Y} \mid \mathbf{R}}(\mathbf{Y}) & =\frac{1}{\pi^{N M} \prod_{i=1}^{N} \lambda_{i}^{M}} e^{-\operatorname{tr} \mathbf{R}^{-1} \mathbf{Y} \mathbf{Y}^{\mathrm{H}}} \\
& =\frac{1}{\pi^{N M} \prod_{i=1}^{N} \lambda_{i}^{M}} e^{-\operatorname{tr} \mathbf{U} \mathbf{\Lambda}^{-1} \mathbf{U}^{\mathrm{H}} \mathbf{Y} \mathbf{Y}^{\mathbf{H}}}
\end{aligned}
$$

where $\mathbf{R}=\mathbf{U} \boldsymbol{\Lambda} \mathbf{U}^{\mathrm{H}}$ for $\mathbf{U} \in \mathbb{C}^{N \times N}$ unitary and $\boldsymbol{\Lambda}$ the diagonal matrix of the eigenvalues of $\mathbf{R}$. We now need a variable change to compute

$$
P_{\mathbf{Y} \mid P_{1}, \ldots, P_{K}}(\mathbf{Y})=\int_{\mathcal{S}_{n}\left(\sigma^{2}\right)} P_{\mathbf{Y} \mid \mathbf{R}, P_{1}, \ldots, P_{K}}(\mathbf{Y}) P_{\mathbf{R} \mid P_{1}, \ldots, P_{K}} d \mathbf{R}
$$

with $\mathcal{S}_{n}\left(\sigma^{2}\right)$ the cone of positive definite complex matrices with smallest $N-n$ eigenvalues equal to $\sigma^{2}$. To this end, consider the one-to-one map

$$
\begin{aligned}
G:(\mathcal{U}(N) / T) \times\left(\sigma^{2}, \infty\right)^{n} & \rightarrow \mathcal{S}_{n}\left(\sigma^{2}\right) \\
\left(\mathbf{U}, \boldsymbol{\Lambda}_{n}\right) & \mapsto \mathbf{R}=\mathbf{U} \boldsymbol{\Lambda} \mathbf{U}^{\mathrm{H}}
\end{aligned}
$$

where $\boldsymbol{\Lambda}=\left(\begin{array}{cc}\boldsymbol{\Lambda}_{n} & 0 \\ 0 & \sigma^{2} \mathbf{I}_{N-n}\end{array}\right)$ with $\mathcal{U}(N) / T$ the space of $N \times$ $N$ unitary matrices with first column composed of real positive entries and $\left(\sigma^{2}, \infty\right)_{\leq}^{n}$ the space of diagonal $n \times n$ matrices with 
non-decreasing real positive diagonal entries greater than $\sigma^{2}$. After variable change, we have

$$
\begin{aligned}
& P_{\mathbf{Y} \mid P_{1}, \ldots, P_{K}}(\mathbf{Y})= \\
& \int P_{\mathbf{Y} \mid G\left(\mathbf{U}, \mathbf{\Lambda}_{n}\right), P_{1}, \ldots, P_{K}}(\mathbf{Y}) P_{G\left(\mathbf{U}, \boldsymbol{\Lambda}_{n}\right) \mid P_{1}, \ldots, P_{K}}|\mathbf{J}(G)| d \mathbf{U} d \mathbf{\Lambda}_{n}
\end{aligned}
$$

with $\mathbf{J}(G)$ the Jacobian matrix of $G$ and the integral is taken over the product space $(\mathcal{U}(N) / T) \times\left(\sigma^{2}, \infty\right)_{<}^{n}$.

For fixed $\mathbf{P}, \mathbf{R}-\sigma^{2} \mathbf{I}_{N}=\mathbf{H} \mathbf{P} \mathbf{H}^{\mathrm{H}}$ is similar to the Wishart matrix $\mathbf{P}^{\frac{1}{2}} \mathbf{H}^{\mathrm{H}} \mathbf{H} \mathbf{P}^{\frac{1}{2}}=\frac{\mathbf{P}^{\frac{1}{2}}}{\sqrt{N}}\left(N \mathbf{H}^{\mathrm{H}} \mathbf{H}\right) \frac{\mathbf{P}^{\frac{1}{2}}}{\sqrt{N}}$; it therefore has the same non-zero eigenvalues. The expression of the ordered non-zero eigenvalue density of $\mathbf{P}^{\frac{1}{2}} \mathbf{H}^{\mathrm{H}} \mathbf{H} \mathbf{P}^{\frac{1}{2}}$, given by [5, Equation (94)], appears to be independent of eigenvalue labeling. This implies that the density of the unordered largest $n$ eigenvalues of $\mathbf{R}$ is merely equal to $1 / n$ ! times their ordered eigenvalue density. Moreover note that the density of $\mathbf{R}$ is invariant by left- and right-product by unitary matrices. The eigenvectors of $\mathbf{R}$ are therefore uniformly distributed over $\mathcal{U}(N)$. This allows us to write

$P_{\mathbf{Y} \mid P_{1}, \ldots, P_{K}}(\mathbf{Y})=$

$\int P_{\mathbf{Y} \mid G^{+}\left(\mathbf{U}, \boldsymbol{\Lambda}_{n}\right), P_{1}, \ldots, P_{K}}(\mathbf{Y}) P_{G^{+}\left(\mathbf{U}, \boldsymbol{\Lambda}_{n}\right) \mid P_{1}, \ldots, P_{K}}|\mathbf{J}(G)| d \mathbf{U} d \mathbf{\Lambda}_{n}$

where we denoted $G^{+}$the extension of $G$ to the surjective function defined on $\mathcal{U}(N) \times\left(\sigma^{2}, \infty\right)^{n}$ and the integral is defined on this space. Since the (Haar) density of $\mathbf{U}$ is uniform over $\mathcal{U}(N)$ and is set here to 1 without loss of generality, we can identify $P_{G^{+}\left(\mathbf{U}, \boldsymbol{\Lambda}_{n}\right) \mid P_{1}, \ldots, P_{K}}\left(\mathbf{U}, \boldsymbol{\Lambda}_{n}\right)|\mathbf{J}(G)|$ with the density $P_{\boldsymbol{\Lambda}_{n} \mid P_{1}, \ldots, P_{K}}\left(\boldsymbol{\Lambda}_{n}\right)$ of $\boldsymbol{\Lambda}_{n}$, to obtain

$P_{\mathbf{Y} \mid P_{1}, \ldots, P_{K}}(\mathbf{Y})=\int P_{\mathbf{Y} \mid \mathbf{U} \mathbf{\Lambda} \mathbf{U}^{H}, P_{1}, \ldots, P_{K}}(\mathbf{Y}) P_{\boldsymbol{\Lambda}_{n} \mid P_{1}, \ldots, P_{K}} d \mathbf{U} d \boldsymbol{\Lambda}_{n}$

the integration being over $\mathcal{U}(N) \times\left(\sigma^{2}, \infty\right)^{n}$.

The random matrix $\boldsymbol{\Lambda}_{n}$ follows the Wishart distribution [5]

$$
\begin{aligned}
& P_{\boldsymbol{\Lambda}_{n} \mid p_{1}, \ldots, p_{n}}\left(\lambda_{1}, \ldots, \lambda_{n}\right)=\frac{\prod_{i=1}^{n} \frac{N^{N}}{p_{i}^{N}} \prod_{i=1}^{n}\left(\lambda_{i}-\sigma^{2}\right)^{N-n}}{n ! \prod_{i=1}^{n}(N-i) !(n-i) !} \\
& \times \prod_{i<j}^{n}\left(\lambda_{j}-\lambda_{i}\right)^{2} \int_{U(n)} e^{-\operatorname{tr} N \mathbf{P}^{-1} \mathbf{U}\left(\mathbf{\Lambda}_{n}-\sigma^{2} \mathbf{I}_{n}\right) \mathbf{U}^{\mathrm{H}}} d \mathbf{U}
\end{aligned}
$$

for $\lambda_{i} \geq \sigma^{2}$. From the Harish-Chandra formula, (Lemma 1, Appendix B), we have

$$
\begin{aligned}
& P_{\Lambda_{n} \mid p_{1}, \ldots, p_{n}}\left(\lambda_{1}, \ldots, \lambda_{n}\right)=\lim _{p_{i} \rightarrow P_{\eta(i)}, \forall i} \frac{N^{n\left(N-\frac{n-1}{2}\right)}}{n !} \\
& \times \prod_{i=1}^{n} \frac{\left(\lambda_{i}-\sigma^{2}\right)^{N-n}}{(N-i) !} \prod_{i<j}^{n}\left(\lambda_{j}-\lambda_{i}\right) \frac{\operatorname{det}\left(e^{-\frac{N\left(\lambda_{j}-\sigma^{2}\right)}{p_{i}}}\right)}{\prod_{i=1}^{n} p_{i}^{N-n+1} \prod_{i<j}^{n}\left(p_{j}-p_{i}\right)}
\end{aligned}
$$

and we are now in position to evaluate the posterior distribution of the $P_{k}$ 's. We present hereafter the final result of the integration in (12) and associated derivations when $n_{k}=1$ for all $k$.

Theorem 1: If $p_{1}, \ldots, p_{n}$ are distinct, then, denoting $\mathbf{y}=$ $\left(y_{1}, \ldots, y_{N}\right)$ the eigenvalues of $\mathbf{Y} \mathbf{Y}^{\mathrm{H}}, P_{\mathbf{Y} \mid p_{1}, \ldots, p_{n}}(\mathbf{Y})$ is given explicitly by (16) below, where $\mathcal{S}_{n}^{N}$ is the set of all permutations of $n$-subsets of $\{1, \ldots, N\}, \mathcal{S}_{n}=\mathcal{S}_{n}^{n},|\mathbf{x}|=\sum_{i} x_{i}, \overline{\mathbf{x}}$ is the complementary of the set $\mathbf{x}, \mathbf{x}[\mathbf{y}]$ is the restriction of $\mathbf{x}$ to the indexes in $\mathbf{y}, \Delta(\mathbf{x})$ is the Vandermonde determinant of $\mathbf{x}$, the constant $C\left(\sigma^{2}\right)$ is given by

$$
C\left(\sigma^{2}\right)=\frac{N^{n\left(M-\frac{n-1}{2}\right)}}{\pi^{N M} n !} \frac{(-1)^{n N+1}}{\sigma^{2(N-n)(M-n)}}
$$

and

$$
\begin{aligned}
J_{k}(x, y) & =\int_{x}^{\infty} u^{k} e^{-u-\frac{y}{u}} d u \\
& =2 y^{\frac{k+1}{2}} K_{-k-1}(2 \sqrt{y})-\int_{0}^{x} u^{k} e^{-u-\frac{y}{u}} d u .
\end{aligned}
$$

The complete derivation of this result is provided in Appendix A. Furthermore, we provide Lemma 2 in Appendix B which can be used to generalize Theorem 16 to the $n_{k} \geq 1$ scenario.

\section{SIMULATION RESULTS}

Figure 1 depicts an example of the posterior probability density (16) for $K=2$ sources and $n_{1}=n_{2}=1$. The peak accurately identifies the true powers, despite the small number of samples and the fact that only the channel statistics are known to the estimator.

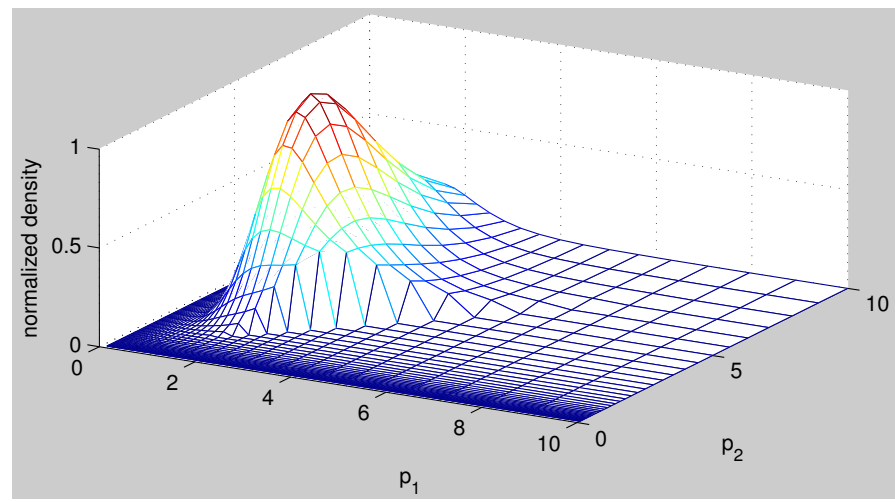

Fig. 1. $P_{\mathbf{Y} \mid P_{1}, P_{2}}$ (normalized) for $M=N=16, \sigma^{2}=0.01$ and a randomly selected channel realization. True $\left(P_{1}, P_{2}\right)=(1,5)$.

We now provide a performance comparison of various estimators of the powers $P_{1}, \ldots, P_{K}$. First, based on Theorem 16, ML and MMSE estimators for $P_{1}, \ldots, P_{K}$ can be derived. Precisely, the joint ML estimator is defined as

$$
\arg \max _{P_{1}, \ldots, P_{K}} P_{\mathbf{Y} \mid P_{1}, \ldots, P_{K}}=\arg \max _{P_{1}, \ldots, P_{K}} P_{P_{1}, \ldots, P_{K} \mid \mathbf{Y}}
$$

while the joint MMSE estimator is given by

$$
\frac{1}{Z} \int\left(P_{1}, \cdots, P_{K}\right) P_{P_{1}, \ldots, P_{K} \mid \mathbf{Y}} d P_{1} \ldots d P_{K}
$$

with $Z=\int P_{P_{1}, \ldots, P_{K} \mid \mathbf{Y}} d P_{1} \ldots d P_{K}$ a normalizing constant. Extensive simulations of the ML and MMSE estimators are however

$$
P_{\mathbf{Y} \mid p_{1}, \ldots, p_{n}}(\mathbf{Y})=\frac{C\left(\sigma^{2}\right) e^{\sum_{i=1}^{n} \frac{N \sigma^{2}}{p_{i}}}}{\prod_{i=1}^{n} p_{i}^{M-n+1} \Delta(\mathbf{p})} \sum_{\mathbf{a} \in S_{n}^{N}}(-1)^{|\mathbf{a}|} \operatorname{sgn}(\mathbf{a}) e^{-\frac{\mid \mathbf{y}[\overline{\mathbf{a}}||}{\sigma^{2}}} \frac{\Delta(\mathbf{y}[\overline{\mathbf{a}}])}{\Delta(\mathbf{y})} \sum_{\mathbf{b} \in S_{n}} \operatorname{sgn}(\mathbf{b}) \prod_{i=1}^{n} J_{N-M-1}\left(\frac{N \sigma^{2}}{p_{b_{i}}}, \frac{N y_{a_{i}}}{p_{b_{i}}}\right)
$$




\begin{tabular}{c|cc} 
& $P_{1}$ & $P_{2}$ \\
\hline Optimum MMSE & 0.1239 & 0.1278 \\
Large dimension & 0.1514 & 0.1332
\end{tabular}

Table I. Normalized mean square error of the estimates.

difficult to obtain due to the necessity of computing several nested numerical integrals. As such, these estimators are only attractive from a theoretical point of view when compared to alternative estimators. In particular, we compare here the performance of the optimal ML and MMSE estimators to a computationally efficient estimator which assumes that all system dimensions are very large and which we introduce hereafter.

In [2], a large dimensional random matrix theory estimator $\left(\hat{P}_{1}, \ldots, \hat{P}_{K}\right)$ of $\left(P_{1}, \ldots, P_{K}\right)$ is derived, which is asymptotically almost surely accurate in the sense that $\hat{P}_{k}-P_{k} \rightarrow 0$ almost surely as $N, n_{1}, \ldots, n_{K}, M \rightarrow \infty$ with finite limiting ratios between all pairs of variables. This relation however holds true under some conditions requiring that the eigenvalues $\boldsymbol{\lambda}=\left(\lambda_{1}, \ldots, \lambda_{N}\right)^{\top}$ of $\frac{1}{N} \mathbf{Y} \mathbf{Y}^{\mathrm{H}}$ are divided in $K$ distinct clusters. This is achieved in particular at high signal to noise ratio, i.e. small values of $\sigma$ compared to $\min _{k} P_{k}$, for $M / N$ rather large and $N / n_{k}$ rather large for each $k$. Under these conditions, the estimator is explicitly given by

$$
\hat{P}_{k}=\frac{N M}{n_{k}(M-N)} \sum_{i \in \mathcal{N}_{k}}\left(\eta_{i}-\mu_{i}\right)
$$

in which $\mathcal{N}_{k}=\left\{N-\sum_{i=k}^{K} n_{i}+1, \ldots, N-\sum_{i=k+1}^{K} n_{i}\right\}$ is the set of indexes matching the cluster of eigenvalues in the spectrum of $\frac{1}{N} \mathbf{Y} \mathbf{Y}^{\mathrm{H}}$ corresponding to $P_{k},\left(\eta_{1}, \ldots, \eta_{N}\right)$ are the ordered eigenvalues of the matrix $\operatorname{diag}(\boldsymbol{\lambda})-\frac{1}{N} \sqrt{\boldsymbol{\lambda}} \sqrt{\boldsymbol{\lambda}}^{\top}$ and $\left(\mu_{1}, \ldots, \mu_{N}\right)$ are the ordered eigenvalues of the matrix $\operatorname{diag}(\boldsymbol{\lambda})-\frac{1}{M} \sqrt{\boldsymbol{\lambda}} \sqrt{\boldsymbol{\lambda}}^{\top}$. A generalization of the formula for $M=N$ can be found in [2].

We consider a the scenario with $K=2$ signal sources with $n_{1}=n_{2}=1, N=M=16$ and $\sigma^{2}=-20 \mathrm{~dB}$. In Figure 2 , the cumulative distribution function (CDF) of 10,000 Monte Carlo simulations for the ML and MMSE estimators against the large dimensional estimator are depicted. The plots suggest that both the ML and MMSE estimators perform similarly and only slightly outperform the large dimensional estimator in the sense that (i) the standard deviations of the individual estimators is smaller, as confirmed by the comparatively steeper CDF around the true powers and (ii) the source separation is higher, as suggested by the more pronounced flattening of the CDF between the true powers. Table I provides comparative normalized mean square error figures of the individual estimates. We notice here that the gap between the optimal and large dimensional methods is not significant. This suggests that the large dimensional estimator is very robust to applications in small dimensional systems, a remark which is often made in the applied research domain of large dimensional random matrix theory [4].

\section{CONCLUSION}

In this article, blind maximum likelihood and minimum mean square error estimators of the joint powers of multiple signal sources are evaluated. These estimators are compared in performance to a recent estimator obtained by large dimensional random matrix analysis. The latter is shown by simulations to perform close to optimally even for small system dimensions, suggesting once

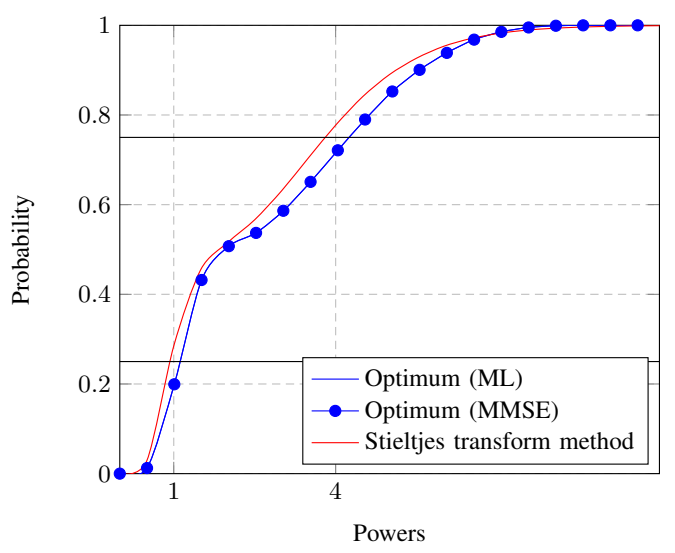

Fig. 2. Distribution function for the detection of two power sources, $P_{1}=1, P_{2}=4$. Optimum against Stieltjes transform method.

again that random matrix theory is an adequate tool for future array processing questions, arising in particular in telecommunications.

\section{ACKNOWLEDGMENT}

This work was supported in parts by the Austrian Science Fund (FWF) through grant NFN SISE (S106), and by the FP7 Newcom++ network of excellence of the European Commission.

\section{APPENDIX A}

\section{Proof OF THEOREM 16}

Recall that, according to the hypotheses of Theorem 16, all $p_{1}, \ldots, p_{n}$ are distinct. From (13), the expression of $P_{\mathbf{Y} \mid p_{1}, \ldots, p_{n}}(\mathbf{Y})$ is

$$
\begin{aligned}
& \int \frac{N^{n\left(N-\frac{n-1}{2}\right)}}{\pi^{N M} \prod_{i=1}^{N} \lambda_{i}^{M}} \frac{e^{-\operatorname{tr} \mathbf{U} \boldsymbol{\Lambda}^{-1} \mathbf{U}^{\mathrm{H}} \mathbf{Y} \mathbf{Y}^{\mathrm{H}}}}{n ! \prod_{i=1}^{n}(N-i) !} \operatorname{det}\left(e^{-\frac{N\left(\lambda_{j}-\sigma^{2}\right)}{p_{i}}}\right) \\
& \times \frac{\prod_{i=1}^{n}\left(\lambda_{i}-\sigma^{2}\right)^{N-n} \prod_{i<j}^{n}\left(\lambda_{j}-\lambda_{i}\right)}{\prod_{i=1}^{n} p_{i}^{N-n+1} \prod_{i<j}^{n}\left(p_{j}-p_{i}\right)} d \mathbf{U} d \boldsymbol{\Lambda} .
\end{aligned}
$$

Applying the Harish-Chandra theorem, (Lemma 1 in Appendix B), this leads after some simplifications to

$$
\begin{gathered}
\int_{\Lambda} \frac{N^{n\left(N-\frac{n-1}{2}\right)} \prod_{i=1}^{N-n-1} i ! \prod_{i=1}^{n}\left(\lambda_{i}-\sigma^{2}\right)^{N-n}}{\pi^{N M} n ! \prod_{i=1}^{N} \lambda_{i}^{M-N+1} \prod_{i=1}^{n} p_{i}^{N-n+1} \prod_{i<j}^{n}\left(p_{j}-p_{i}\right)} \\
\quad \times \frac{\operatorname{det}\left(e^{-\frac{N\left(\lambda_{j}-\sigma^{2}\right)}{p_{i}}}\right)_{n} \operatorname{det}\left(e^{-\frac{y_{i}}{\lambda_{j}}}\right)_{N}}{\prod_{i<j}^{N}\left(y_{j}-y_{i}\right)} d \boldsymbol{\Lambda}
\end{gathered}
$$

where the index $n$ in $\operatorname{det}(\mathbf{X})_{n}$ is here to recall the size of the underlying matrix.

At this point, we realize that both extreme right numerator and denominator are null since the smallest $N-n$ eigenvalues of $\lambda_{n+1}, \ldots, \lambda_{N}$ are all equal to $\sigma^{2}$. In order to solve this indeterminate form, we then use [6, Lemma 6], to obtain, after simplification

$$
\begin{gathered}
P_{\mathbf{Y} \mid p_{1}, \ldots, p_{n}}(\mathbf{Y})=\int_{\Lambda} \frac{N^{n\left(N-\frac{n-1}{2}\right)} \operatorname{det}\left(e^{-\frac{N\left(\lambda_{j}-\sigma^{2}\right)}{p_{i}}}\right)_{n}}{\pi^{N M} n ! \sigma^{2(M-N+1)(N-n)} \prod_{i=1}^{n} \lambda_{i}^{M-N+1}} \\
\times \frac{\operatorname{det}\left[e^{-\frac{y_{i}}{\sigma^{2}}}, \ldots,\left(\frac{y_{i}^{N-n-1}}{\sigma^{4(N-n-1)}}+\ldots\right) e^{-\frac{y_{i}}{\sigma^{2}}}, e^{-\frac{y_{i}}{\lambda_{1}}}, \ldots, e^{-\frac{y_{i}}{\lambda n}}\right]}{\prod_{i=1}^{n} p_{i}^{N-n+1} \prod_{i<j}^{n}\left(p_{j}-p_{i}\right) \prod_{i<j}^{N}\left(y_{j}-y_{i}\right)} d \boldsymbol{\Lambda}
\end{gathered}
$$


which is well defined since all $p_{i}$ 's are different. If they were not, Lemma 2 would be needed, which leads to a rather lengthy expression which we do not further discuss here for simplicity. We mention that the notation '.. ' in $\left(\frac{y_{i}^{N-n-1}}{\sigma^{4(N-n-1)}}+\ldots\right) e^{-\frac{y_{i}}{\sigma^{2}}}$ indicates that the extra terms are polynomials of the variable $y_{i}$ of degree less than $y_{i}^{N-n-1}$. It is clear that linear combinations of the first columns allows one to discard all these extra terms and therefore we can consider the matrix that only has one leading coefficient $y_{i}^{k}$ in the entries of column $k$.

Extracting the successive powers of $\sigma$ which multiply each column and then the successive terms $e^{-\frac{y_{i}}{\sigma^{2}}}$ which multiply each row, we therefore further have

$P_{\mathbf{Y} \mid p_{1}, \ldots, p_{n}}(\mathbf{Y})=\int_{\Lambda} \frac{N^{n\left(N-\frac{n-1}{2}\right)}}{\pi^{N M} n !} \frac{\operatorname{det}\left(e^{-\frac{N\left(\lambda_{j}-\sigma^{2}\right)}{p_{i}}}\right)_{n} e^{-\frac{|\mathbf{y}|}{\sigma^{2}}}}{\sigma^{2(N-n)(M-n)} \prod_{i=1}^{n} \lambda_{i}^{M-N+1}}$

$\times \frac{\operatorname{det}\left[1, y_{i}, \ldots, y_{i}^{N-n-1}, e^{-\left(\frac{y_{i}}{\lambda_{1}}-\frac{y_{i}}{\sigma^{2}}\right)}, \ldots, e^{-\left(\frac{y_{i}}{\lambda_{n}}-\frac{y_{i}}{\sigma^{2}}\right)}\right]}{\prod_{i=1}^{n} p_{i}^{N-n+1} \prod_{i<j}^{n}\left(p_{j}-p_{i}\right) \prod_{i<j}^{N}\left(y_{j}-y_{i}\right)} d \boldsymbol{\Lambda}$.

We now develop the second determinant successively along its last $n$ columns. Noticing that the square matrix formed of the first $N-n$ columns with any missing rows $a_{1}, \ldots, a_{n}$ is a Vandermonde matrix with determinant $\Delta(\mathbf{y}[\overline{\mathbf{a}}])=\prod_{i, j \notin\left\{a_{1}, \ldots, a_{n}\right\}}\left(y_{j}-y_{i}\right)$, we obtain

$$
\begin{aligned}
& \operatorname{det}\left[1, y_{i}, \ldots, y_{i}^{N-n-1}, e^{-\left(\frac{y_{i}}{\lambda_{1}}-\frac{y_{i}}{\sigma^{2}}\right)}, \ldots, e^{-\left(\frac{y_{i}}{\lambda_{n}}-\frac{y_{i}}{\sigma^{2}}\right)}\right] \\
& =\sum_{\mathbf{a} \in \mathcal{S}_{n}^{N}}(-1)^{\left|a_{i}\right|+N n+1} \operatorname{sgn}(\mathbf{a}) \Delta(\mathbf{y}[\mathbf{\mathbf { a }}]) \frac{e^{-\sum_{i=1}^{n} \frac{y_{a_{i}}}{\lambda_{i}}} e^{\frac{1}{\sigma^{2}} \sum_{i=1}^{n} y_{a_{i}}}}{\Delta(\mathbf{y})}
\end{aligned}
$$

with $\mathcal{S}_{n}^{N}, n \leq N$, the set of all permutations of the $n$-subsets of $\{1, \ldots, N\}$. The factor $(-1)^{\left|a_{i}\right|+N n+1}$ is linked to the determinant expansion formula and is obtained when considering the different row indexations resulting from successive removals of rows from the main $N \times N$ matrix.

It remains to express the first determinant of (25) under the form of products of functions of the $\lambda_{i}$ 's. This unfolds directly by writing

$$
\begin{aligned}
\operatorname{det}\left(e^{\left.-\frac{N\left(\lambda_{j}-\sigma^{2}\right)}{p_{i}}\right)_{n}}\right. & =e^{-N \sigma^{2} \sum_{i=1}^{n} \frac{1}{p_{i}}} \operatorname{det}\left(e^{-N \frac{\lambda_{j}}{p_{i}}}\right)_{n} \\
& =e^{-N \sigma^{2} \sum_{i=1}^{n} \frac{1}{p_{i}}} \sum_{\mathbf{b} \in \mathcal{S}_{n}} \operatorname{sgn}(\mathbf{b}) \prod_{i=1}^{n} e^{-N \frac{y_{i}}{p_{b_{i}}}}
\end{aligned}
$$

with $\mathcal{S}_{n}=\mathcal{S}_{n}^{n}$.

Gathering those results together, we end up with

$$
\begin{aligned}
& P_{\mathbf{Y} \mid p_{1}, \ldots, p_{n}}(\mathbf{Y})=\frac{N^{n\left(N-\frac{n-1}{2}\right)}}{\pi^{N M} n !} \frac{(-1)^{n N+1}}{\sigma^{2(N-n)(M-n)}} \frac{e^{N \sigma^{2} \sum_{i=1}^{n} \frac{1}{p_{i}}}}{\prod_{i=1}^{n} p_{i}^{N-n+1} \Delta(\mathbf{p})} \\
& \times \sum_{\mathbf{a} \in \mathcal{S}_{n}^{N}}(-1)^{|\mathbf{a}|} \operatorname{sgn}(\mathbf{a}) e^{-\frac{|\mathbf{y}[\overline{\mathbf{a}}]|}{\sigma^{2}}} \frac{\Delta(\mathbf{y}[\overline{\mathbf{a}}])}{\Delta(\mathbf{y})} \\
& \times \sum_{\mathbf{b} \in \mathcal{S}_{n}} \operatorname{sgn}(\mathbf{b}) \prod_{i=1}^{n} \int_{\sigma^{2}}^{\infty} \lambda_{i}^{N-M-1} e^{-\frac{N y_{i}}{p_{b_{i}}}-\frac{y a_{i}}{\lambda_{i}}} d \lambda_{i} .
\end{aligned}
$$

With the change of variable $u=N y_{i} / p_{b_{i}}$, the integral above becomes

$\int_{\sigma^{2}}^{\infty} \lambda_{i}^{N-M-1} e^{-\frac{N y_{i}}{p_{b_{i}}}-\frac{y a_{i}}{\lambda_{i}}} d \lambda_{i}=\frac{N^{M-N}}{p_{b_{i}}^{M-N}} J_{N-M-1}\left(\frac{N \sigma^{2}}{p_{b_{i}}}, \frac{N y_{a_{i}}}{p_{b_{i}}}\right)$.

Inserting the factor $\frac{N^{M-N}}{p_{b_{i}}^{M-N}} J_{N-M-1}$ in the expression of $P_{\mathbf{Y} \mid p_{1}, \ldots, p_{n}}(\mathbf{Y})$, we finally obtain (16).

\section{APPENDIX B \\ LEMMAS}

Lemma 1 ([7]): For non singular $N \times N$ positive definite Hermitian matrices $\mathbf{A}$ and $\mathbf{B}$ of respective eigenvalues $a_{1}, \ldots, a_{N}$ and $b_{1}, \ldots, b_{N}$, such that for all $i \neq j, a_{i} \neq a_{j}$ and $b_{i} \neq b_{j}$, we have

$$
\begin{aligned}
\int_{\mathbf{U} \in \mathcal{U}(N)} e^{\kappa \operatorname{tr}\left(\mathbf{A U B U}^{\mathbf{H}}\right)} d \mathbf{U} \\
=\left(\prod_{i=1}^{N-1} i !\right) \kappa^{\frac{1}{2} N(N-1)} \frac{\operatorname{det}\left(\left\{e^{-b_{j} a_{i}}\right\}_{1 \leq i, j \leq N}\right)}{\Delta(\mathbf{A}) \Delta(\mathbf{B})}
\end{aligned}
$$

where, for any bivariate function $f,\{f(i, j)\}_{1 \leq i, j \leq N}$ denotes the $N \times N$ matrix of $(i, j)$ entry $f(i, j)$, and $U(N)$ is the space of $N \times N$ unitary matrices.

Lemma 2 (Generalization of [6, Lemma 6]): Let $f_{1}, \ldots, f_{N}$ be a family of infinitely differentiable functions and let $x_{1}, \ldots, x_{N} \in \mathbb{R}$. Denote

$$
R\left(x_{1}, \ldots, x_{N}\right) \triangleq \frac{\operatorname{det}\left(\left\{f_{i}\left(x_{j}\right)\right\}_{i, j}\right)}{\prod_{i<j}\left(x_{j}-x_{i}\right)} .
$$

Then, for $N_{1}, \ldots, N_{p}$ such that $N_{1}+\ldots+N_{p}=N$ and for $y_{1}, \ldots, y_{p} \in$ $\mathbb{R}$ distinct

$$
\begin{aligned}
& \lim _{x_{1}, \ldots, x_{N_{1}} \rightarrow y_{1}} R\left(x_{1}, \ldots, x_{N}\right) \\
& x_{N-N_{p}+1}, \ldots, x_{N} \rightarrow y_{p} \\
& =\frac{\operatorname{det}\left[f_{i}\left(y_{1}\right), \ldots, f_{i}^{\left(N_{1}-1\right)}\left(y_{1}\right), \cdots, f_{i}\left(y_{p}\right), \ldots, f_{i}^{\left(N_{p}-1\right)}\left(y_{p}\right)\right]}{\prod_{1 \leq i<j \leq p}\left(y_{j}-y_{i}\right)^{N_{i} N_{j}} \prod_{l=1}^{p} \prod_{j=1}^{N_{l}-1} j !} .
\end{aligned}
$$

The proof, by induction, following the technique outlined in [6, Lemma $6]$, is omitted here.

\section{APPENDIX C \\ REFERENCES}

[1] X. Mestre, "Improved estimation of eigenvalues of covariance matrices and their associated subspaces using their sample estimates," IEEE Trans. Inf. Theory, vol. 54, no. 11, pp. 5113-5129, Nov. 2008.

[2] R. Couillet, J. W. Silverstein, and M. Debbah, "Eigen-Inference for Energy Estimation of Multiple Sources," IEEE Trans. Inf. Theory, 2011, to appear. [Online]. Available: http://arxiv.org/abs/1001.3934

[3] J. Yao, R. Couillet, J. Najim, E. Mouline, and M. Debbah, "CLT for eigen-inference methods in cognitive radios," in Proc. IEEE International Conference on Acoustics, Speech and Signal Processing (ICASSP'11), Prague, Czech Republic, 2011, submitted for publication.

[4] R. Couillet and M. Debbah, Random matrix methods for wireless communications, 1st ed. New York, NY, USA: Cambridge University Press, 2011, to appear.

[5] A. T. James, "Distributions of matrix variates and latent roots derived from normal samples," The Annals of Mathematical Statistics, vol. 35, no. 2, pp. 475-501, 1964.

[6] S. H. Simon, A. L. Moustakas, and L. Marinelli, "Capacity and character expansions: Moment generating function and other exact results for MIMO correlated channels," IEEE Trans. Inf. Theory, vol. 52, no. 12, pp. 5336-5351, 2006.

[7] Harish-Chandra, "Differential operators on a semi-simple Lie algebra," American Journal of Mathematics, vol. 79, pp. 87-120, 1957. 